\title{
Local rigidity of Einstein 4-manifolds satisfying a chiral curvature condition
}

\author{
Joel Fine $^{* 1}$, Kirill Krasnov ${ }^{2}$, and Michael Singer ${ }^{3}$ \\ ${ }^{1}$ Université libre de Bruxelles, Belgium \\ ${ }^{2}$ University of Nottingham, UK \\ ${ }^{3}$ University College London, UK
}

June 3, 2020

\begin{abstract}
Let $(M, g)$ be a compact oriented Einstein 4-manifold. Write $R_{+}$for the part of the curvature operator of $g$ which acts on self-dual 2 -forms. We prove that if $R_{+}$is negative definite then $g$ is locally rigid: any other Einstein metric near to $g$ is isometric to it. This is a chiral generalisation of Koiso's Theorem, which proves local rigidity of Einstein metrics with negative sectional curvature. Our hypotheses are roughly one half of Koiso's. Our proof uses a new variational description of Einstein 4-manifolds, as critical points of the so-called pure connection action $S$. The key step in the proof is that when $R_{+}<0$, the Hessian of $S$ is strictly positive modulo gauge.
\end{abstract}

\section{Contents}

1 Introduction 2

1.1 Statement of the main result . . . . . . . . . . . . . . . . . . . . . . . . . . 2

1.2 Outline of the proof . . . . . . . . . . . . . . . . . . . . . . . . . . . . . . . . . .

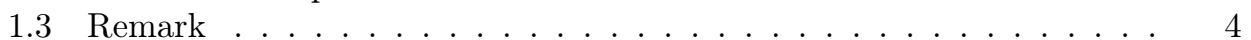

2 From Plebanski to the pure connection action 4

2.1 Some foundational 4-dimensional facts . . . . . . . . . . . . . . 4

2.2 The Plebanski action . . . . . . . . . . . . . . . . . . . . . . 5

2.3 The pure connection action . . . . . . . . . . . . . . . . . 6

2.4 The gauge group . . . . . . . . . . . . . . . . . . . . 8

3 The Hessian of the pure connection action 8

3.1 The Hessian before gauge fixing . . . . . . . . . . . . . . . . . . . . . . . . 9

3.2 The Hessian after gauge fixing . . . . . . . . . . . . . . . . . . . . . . 10

3.3 Gauge fixing . . . . . . . . . . . . . . . . . . . . . . . . . . . . . . . . . . . . . . 11

3.4 The proof of Theorem 3.1 . . . . . . . . . . . . . . . . . . . . . . . . . 14

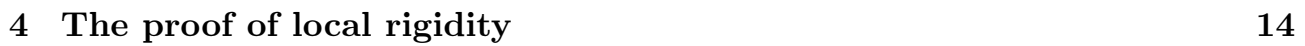

*JF was supported by ERC consolidator grant 646649 "SymplecticEinstein" and EoS grant 30950721. 


\section{Introduction}

\subsection{Statement of the main result}

A Riemannian metric $g$ is called Einstein if its Ricci curvature is constant:

$$
\operatorname{Ric}(g)=\Lambda g, \quad \Lambda \in \mathbb{R}
$$

If $g$ is Einstein and $\phi$ is a diffeomorphism, then $\phi^{*} g$ is also Einstein. When considered modulo diffeomorphisms however equation $(1.1)$ is elliptic of index zero. This means that naively one might expect Einstein metrics to be isolated modulo diffeomorphisms, or "locally rigid". It is important to note that there are many situations in which Einstein metrics are not rigid: hyperbolic metrics on surfaces, many Kähler-Einstein metrics, holonomy $G_{2}$ metrics, ... The aim of this article is to give a curvature condition in dimension 4 which ensures this naive expectation of local rigidity is true.

We begin with the definition.

\section{Definition 1.1.}

1. Given an Einstein metric $g$, let $D_{g}$ denote the linearisation of the map $g \mapsto$ $\operatorname{Ric}(g)-\Lambda g$ at $g$. An infinitesimal Einstein deformation of $g$ is a section $h$ of $S^{2} T^{*} M$ for which $D_{g}(h)=0$.

2. An Einstein metric $g$ is called locally rigid if for any infinitesimal Einstein deformation $h$ of $g$ there is a vector field $v$ such that $h=L_{v} g$.

Let $(M, g)$ be a locally rigid compact Einstein metric. It can be shown that if $\widehat{g}$ is another Einstein metric sufficiently close to $g$ (in an appropriate Hölder space, say) then there is a diffeomorphism $\phi$, close to the identity, with $\hat{g}=\phi^{*} g$. (For a proof see, for example, 2.) This justifies the use of the term "local rigidity" rather than just "infinitesimal rigidity" in the preceding definition.

To state our local rigidity result, we first recall the decomposition of the curvature tensor of an oriented Riemannian 4-manifold $\left(M^{4}, g\right)$. In this dimension the 2 -forms split $\Lambda^{2}=\Lambda^{+} \oplus \Lambda^{-}$as the \pm 1 -eigenspaces of the Hodge star. This induces a decomposition of the curvature operator:

$$
\mathrm{Rm}=\left(\begin{array}{cc}
R_{+} & C^{*} \\
C & R_{-}
\end{array}\right)
$$

Here $R_{+} \in \operatorname{End}\left(\Lambda^{+}\right)$and $R_{-} \in \operatorname{End}\left(\Lambda^{-}\right)$are self-adjoint, whilst $C \in \operatorname{Hom}\left(\Lambda^{+}, \Lambda^{-}\right)$. This relates to the standard decomposition into the scalar, trace-free Ricci and Weyl curvatures (denoted $R, \operatorname{Ric}^{0}$ and $W$ respectively) as follows: $R=4 \operatorname{Tr}\left(R_{+}\right)=4 \operatorname{Tr}\left(R_{-}\right)$, Ric $^{0}=C$, and $W=R_{+}^{0} \oplus R_{-}^{0}$ (where $E^{0}$ denotes the trace-free part of $E$ and where we have used a suitable identification of $\operatorname{Hom}\left(\Lambda^{+}, \Lambda^{-}\right)$with symmetric trace-free bilinear forms on $T M$ ). In particular, when $g$ is Einstein, $\mathrm{Rm}=R_{+} \oplus R_{-}$.

We now state our main result. In the statement the expression $R_{+}<0$ means that the quadratic form $\left(R_{+}(w), w\right)$ is negative definite.

Theorem 1.2. Let $g$ be an Einstein metric on a compact orientable 4-manifold, with either $R_{+}<0$ or $R_{-}<0$. Then $g$ is locally rigid.

To put our main result in context, we first describe one situation in which local rigidity has already been established, namely Koiso's Theorem.

Theorem 1.3 (Koiso [7). Let $g$ be an Einstein metric on a compact orientable manifold of dimension at least 3, with negative curvature (i.e. all sectional curvatures of $g$ are negative). Then $g$ is locally rigid.

Theorem 1.2 is a chiral version of Koiso's Theorem, in dimension 4, with roughly "one half" the hypotheses. To see this, we relate the sectional curvatures to $R_{+}$and $R_{-}$. Let $u, v$ be orthogonal unit tangent vectors, write $u^{b}, v^{b}$ for their metric dual 
covectors and $\alpha=\left(u^{b} \wedge v^{b}\right)^{+}$and $\beta=\left(u^{b} \wedge v^{b}\right)^{-}$. When $g$ is Einstein the sectional curvature in the plane spanned by $u$ and $v$ is

$$
\sec (u, v)=\left\langle R_{+}(\alpha), \alpha\right\rangle+\left\langle R_{-}(\beta), \beta\right\rangle
$$

By letting $u, v$ vary, we can make $\alpha$ and $\beta$ take any values in the spheres in $\Lambda^{+}$and $\Lambda^{-}$. Write $\lambda$ for the largest eigenvalue of $R_{+}$and $\mu$ for the largest eigenvalue of $R_{-}$. The requirement that an Einstein metric have negative sectional curvatures is then equivalent to $\lambda+\mu<0$, whilst the hypothesis in our main result, Theorem 1.2 , is that either $\lambda<0$ or $\mu<0$.

We remark that global rigidity results are much more difficult to establish. The only known results are the following: if $(M, g)$ is a compact hyperbolic or complexhyperbolic 4-manifold then $g$ is the unique Einstein metric on $M$ up to scale and diffeomorphism. In the hyperbolic case this is due to Besson-Courtois-Gallot [1, whilst the complex-hyperbolic case is due to LeBrun [9].

\subsection{Outline of the proof}

Consider first Koiso's Theorem. To prove local rigidity of a negatively curved Einstein metric, Koiso uses a clever Bochner trick. Given an infinitesimal Einstein variation $h$, it is possible to find a vector field $v$ so that $h-L_{v} g$ solves an equation of the form $\mathscr{D}\left(h-L_{v} g\right)=0$ where $\mathscr{D}$ is a Laplace type operator. Koiso finds a Weitzenböck formula $\mathscr{D}=d^{*} d+B$, where $d$ is an appropriate first order operator. Crucially the remainder term $B$ is positive when the sectional curvatures of $g$ are negative. It follows that $h=L_{v} g$.

Our proof of local rigidity is different. We use a special variational formulation of 4-dimensional Einstein metrics, due to the second author [8] and independently, albeit in a weaker form, to the first author [3]. A full description of this "pure connection formalism" which is accessible to mathematicians appears in [5. We give a brief summary here and more details in $\$ 2$, choosing a description which is better suited to our purposes here than that given in the above references.

Let $E \rightarrow M^{4}$ be an $\mathrm{SO}(3)$-vector bundle over $M$. The following definition is crucial to what follows. It first appeared in this form in [4, but is actually a special case of Weinstein's "fat connections" [12].

Definition 1.4. An $\mathrm{SO}(3)$-connection $A$ on $E$ is called definite if the curvature $F_{A}(u, v)$ is non-zero whenever $u$ and $v$ are linearly independent tangent vectors on $M$.

Examples can be found from Riemannian geometry. Let $(M, g)$ be an oriented Riemannian 4-manifold, take $E=\Lambda^{+}$and $A$ to be the Levi-Civita connection of $g$. Then $A$ is definite precisely when $R_{+}^{2}-C^{*} C$ is a definite endomorphism of $\Lambda^{+}$(we use here the notation of the curvature decomposition 1.2 ; the proof that these metrics give definite connections is in [4). Notice that Einstein metrics have $C=0$ and so when $R_{+}$is also non-degenerate (all eigenvalues are non-zero) these metrics give definite connections. Examples include the constant curvature metrics on hyperbolic space or the four-sphere or, more generally, anti-self-dual Einstein metrics with nonzero scalar curvature.

When $A$ is definite it determines in a canonical way a Riemannian metric $g_{A}$. The metric tensor $g_{A}$ is built pointwise out of the curvature $F_{A}$. The precise formula for $g_{A}$ is given below in $\$ 2$ but it is not important for this introductory discussion. We note however that if $A$ is the Levi-Civita connection on $\Lambda^{+}$for a Riemannian metric $g$ as in the previous paragraph, then it is not in general true that $g_{A}=g$. This happens precisely when $g$ is Einstein and $R_{+}$is definite, with all eigenvalues nonzero and having the same sign.

Write $S(A)=\frac{\Lambda}{2} \int_{M} \operatorname{dvol}\left(g_{A}\right)$ where $\operatorname{dvol}\left(g_{A}\right)$ is the volume form of the metric $g_{A}$. The key fact we need is: 
Theorem 1.5 ([8], see also [5]). If $A$ is a critical point for $S$ then $g_{A}$ is an Einstein metric, with $R_{+}$a definite endomorphism of $\Lambda^{+}$. Conversely, all such Einstein metrics arise this way, with $A$ corresponding to the Levi-Civita connection on $\Lambda^{+}$.

We now return to the proof of Theorem 1.2 Let $g$ be an Einstein metric for which $R_{+}<0$. (Note that reversing the orientation of $M$ swaps $R_{+}$and $R_{-}$and so when proving Theorem 1.2 it suffices to consider $R_{+}<0$.) The Levi-Civita connection of $g$ on $E=\Lambda^{+}$gives a critical point of $S$. The key to proving Theorem 1.2 is to show that $R_{+}<0$ ensures that the Hessian of $S$ at $A$ is strictly positive modulo gauge. So $A$ is an isolated local minimum of $S$ (again, modulo gauge). We then show that this implies the original Einstein metric $g$ is locally rigid.

The article is laid out as follows. In $\$ 2$ we describe the pure connection formalism, showing how to derive it from the Plebanski formulation of Einstein's equations. We then use this derivation in $\$ 3$ to compute the Hessian of the pure connection action. $\$ 4$ uses this to prove local rigidity of $g$.

\subsection{Remark}

We hope this proof highlights an important difference between the pure connection action $S$ and the more classical variational approach to Einstein metrics, given by the Einstein-Hilbert action $S_{\mathrm{EH}}$. At a critical point, the Hessian of $S_{\mathrm{EH}}$ is always indefinite, having an infinite number of both positive and negative eigenvalues. This makes $S_{\mathrm{EH}}$ extremely difficult to analyse from the point of view of the calculus of variations. The pure connection action does not suffer from this problem. Irrespective of curvature assumptions, at a critical point its Hessian is elliptic (modulo gauge), with at most finitely many negative eigenvalues [5]. It remains to be seen if techniques from the calculus of variations can be used to greater effect with the pure connection action than has been possible for the Einstein-Hilbert action.

\section{From Plebanski to the pure connection action}

In [10] Plebanski gave an alternative formulation of Einstein 4-manifolds, as critical points of a certain functional, now called the Plebanski action. By "integrating out" some of the variables of the Plebanski action, one arrives at the pure connection action of [8]. This point of view is central to our computation of the Hessian of the pure connection action in $\$ 3$

Before describing Plebanski's action, we first recall some basic facts about 4dimensional Riemannian geometry, with an emphasis which, whilst it may seem unusual at first sight, is well suited to our later purposes.

\subsection{Some foundational 4-dimensional facts}

Let $M$ be an oriented 4-manifold. The wedge product on 2 -forms $\Lambda^{2} \otimes \Lambda^{2} \rightarrow \Lambda^{4}$ is a non-degenerate symmetric bilinear form on $\Lambda^{2}$ with values in $\Lambda^{4}$. We interpret this as a fibrewise conformal structure on $\Lambda^{2}$ with signature $(3,3)$. Given any Riemannian metric $g$ on $M$, this inner product makes self-dual and anti-self-dual forms orthogonal, is positive definite on $\Lambda_{g}^{+}$and negative definite on $\Lambda_{g}^{-}$. Conversely, given any 3dimensional sub-bundle $P \subset \Lambda^{2}$ on which the wedge-product is positive definite, there is a unique conformal class of metrics $[g]$ on $M$ for which $P=\Lambda_{g}^{+}$. A Riemannian metric is thus equivalent to the data of a positive definite sub-bundle $P$ together with a choice of volume form.

The abstract isomorphism class of the vector bundle $\Lambda_{g}^{+}$is independent of $g$; they are all isomorphic to some fixed reference $\mathrm{SO}(3)$-bundle $E \rightarrow M$. We fix this choice of $E$ once and for all. For any Riemannian metric $g$ on $M$, there is a (non-unique) vector bundle homomorphism $\Sigma: E \rightarrow \Lambda^{2}$ which is an isometry onto $\Lambda_{g}^{+}$. In fact it is convenient to fix the scale so that unit-length vectors in $E$ are sent to self-dual 2-forms 
of length $\sqrt{2}$. The metric $g$ can be recovered from $\Sigma$ by setting $\Lambda^{+}(g)=\Sigma(E)$ and taking the volume form $\mu=\frac{1}{2} \Sigma(e) \wedge \Sigma(e)$ where $e$ is any unit-length element of $E$. This leads us to the following definition.

Definition 2.1. $\Sigma \in \Omega^{2}\left(M, E^{*}\right)$ is called wedge-orthogonal if when thought of as a vector bundle homomorphism $\Sigma: E \rightarrow \Lambda^{2}$ there is a positive 4 -form $\mu$ such that for any pair $e_{1}, e_{2} \in E$, we have $\Sigma\left(e_{1}\right) \wedge \Sigma\left(e_{2}\right)=2\left\langle e_{1}, e_{2}\right\rangle \mu$.

Given a wedge-orthogonal $\Sigma$, there is a unique Riemannian metric $g_{\Sigma}$ on $M$ for which $\Sigma$ takes values in $\Lambda_{g_{\Sigma}}^{+}$and with $\operatorname{dvol}\left(g_{\Sigma}\right)=\mu$.

In the Plebanski formulation, we will use wedge-orthogonal 2-forms to parametrise metrics. Next we need to describe the Levi-Civita connection in this picture. For a proof of the following Lemma see Lemmas 2.3 and 2.4 of [3].

Lemma 2.2. Let $\Sigma \in \Omega^{2}\left(M, E^{*}\right)$ be wedge-orthogonal. There is a unique metric connection $A$ in $E$ for which $\mathrm{d}_{A} \Sigma=0$. This connection is the pull-back to $E$ of the Levi-Civita connection in $\Lambda_{g_{\Sigma}}^{+}$via the isomorphism $\Sigma: E \rightarrow \Lambda_{g_{\Sigma}}^{+}$.

The equation $\mathrm{d}_{A} \Sigma=0$ is a torsion-free condition, see [3] for more details. We are now in a position to recognise Einstein metrics in this set-up. The following result is the translation of the familiar fact that a Riemannian 4-manifold is Einstein if and only if the Levi-Civita connection on $\Lambda^{+}$is a self-dual instanton.

Lemma 2.3. Let $\Sigma \in \Omega^{2}\left(M, E^{*}\right)$ be wedge-orthogonal, with associated metric $g_{\Sigma}$ and let $A$ be the unique metric connection in $E$ with $\mathrm{d}_{A} \Sigma=0$. Then $g_{\Sigma}$ is Einstein if and only if $F_{A}$ is self-dual, i.e. $A$ is an instanton with respect to $g_{\Sigma}$.

\subsection{The Plebanski action}

The Plebanski action is a function of three variables, $(A, \Sigma, \Psi)$ where $A$ is a metric connection on the bundle $E, \Sigma \in \Omega^{2}\left(M, E^{*}\right)$ and $\Psi \in \Omega^{0}\left(M, S_{0}^{2} E\right)$. Note at this stage these variables are arbitrary; in particular, $\Sigma$ is not assumed to be wedge-orthogonal and is unrelated to $A$.

To describe the action, it is perhaps simplest to choose a local orthonormal frame $e_{i}$ for $E$. Pick an orientation on $E$ and take the local frame also to be oriented (the freedom in choice of orientation will turn out to be slightly subtle - we will return to this point later). We write $e^{i}$ for the dual frame of $E^{*}$ and $\widehat{e}^{i}$ for the generator of positive rotations about $e_{i}$, giving a local frame of $\mathfrak{s o}(E)$. We use the convention that repeated indices are summed over $1,2,3$.

We write the connection $A$ locally as

$$
\mathrm{d}_{A}\left(e_{i}\right)=\epsilon_{i j k} A^{j} \otimes e^{k}
$$

for a triple of 1-forms $A^{j}$. Similarly, we write $\Sigma=\Sigma_{i} \otimes e^{i}$ for a triple of 2-forms $\Sigma_{i}$ and $\Psi=\Psi^{i j} e_{i} \otimes e_{j}$ for a symmetric trace-free matrix valued function $\Psi_{i j}$. We will also need the local expression for the curvarure of $A$. Let

$$
F^{i}=\mathrm{d} A^{i}-\frac{1}{2} \epsilon_{j k}^{i} A^{j} \wedge A^{k}
$$

These are the curvature 2-forms, meaning that $\left(\mathrm{d}_{A}\right)^{2}\left(e_{i}\right)=\epsilon_{i j}{ }^{k} F^{j} \otimes e_{k}$.

Definition 2.4. Fix $\Lambda \in \mathbb{R}$. The Plebanski action is the functional

$$
S_{P}(A, \Sigma, \Psi)=\int_{M}\left\{F^{i} \wedge \Sigma_{i}-\frac{1}{2}\left(\Psi^{i j}+\frac{\Lambda}{3} \delta^{i j}\right) \Sigma_{i} \wedge \Sigma_{j}\right\}
$$

(Note that the integrand does not depend on the choice of local frame $e_{i}$ and so makes sense globally.)

Write $\mathscr{P}$ for the open set of triples $(A, \Sigma, \Psi)$ for which $\operatorname{Tr}(\Sigma \wedge \Sigma)>0$. 
Theorem 2.5 (Plebanski [10]). $(A, \Sigma, \Psi) \in \mathscr{P}$ is a critical point of $S_{P}$ if and only if $\Sigma$ is wedge-orthogonal and the associated metric $g_{\Sigma}$ is Einstein.

Proof. Let $a \in \Omega^{1}(M, \mathfrak{s o}(E)), \phi \in \Omega^{0}\left(M, S^{2} E_{0}\right)$ and $\sigma \in \Omega^{2}\left(M, E^{*}\right)$. We compute the partial derivatives of $S_{P}$ in these directions.

$$
\begin{aligned}
& \frac{\partial S_{P}}{\partial A}(a)=\int_{M}\left(\mathrm{~d}_{A} a\right)^{i} \wedge \Sigma_{i}=\int_{M} a^{i} \wedge\left(\mathrm{d}_{A} \Sigma\right)_{i} \\
& \frac{\partial S_{P}}{\partial \Psi}(\phi)=-\frac{1}{2} \int_{M} \phi^{i j} \Sigma_{i} \wedge \Sigma_{j} \\
& \frac{\partial S_{P}}{\partial \Sigma}(\sigma)=\int_{M}\left\{F^{i} \wedge \sigma_{i}-\left(\Psi^{i j}+\frac{\Lambda}{3} \delta^{i j}\right) \Sigma_{i} \wedge \sigma_{j}\right\}
\end{aligned}
$$

From this it follows that

$$
\begin{aligned}
& \frac{\partial S_{P}}{\partial A}=0 \Longleftrightarrow \mathrm{d}_{A} \Sigma=0 \\
& \frac{\partial S_{P}}{\partial \Psi}=0 \Longleftrightarrow \Sigma \text { is wedge-orthogonal } \\
& \frac{\partial S_{P}}{\partial \Sigma}=0 \Longleftrightarrow F^{i}=\left(\Psi^{i j}+\frac{\Lambda}{3} \delta^{i j}\right) \Sigma_{j}
\end{aligned}
$$

By (2.7), $\Sigma$ determines a metric $g_{\Sigma}$, with $\Lambda^{+}=\operatorname{im} \Sigma$, the image of $\Sigma$. By (2.6) and Lemma 2.2 the connection $A$ is the pull-back via $\Sigma$ of the Levi-Civita connection. By 2.8, $F_{A}$ is self-dual. The result follows from Lemma 2.3

When $(A, \Sigma, \Psi)$ is a critical point of $S_{P}$, equation 2.8 tells us that the Einstein metric $g_{\Sigma}$ has $R_{+}=\Psi+\frac{\Lambda}{3}$ Id. In other words, the self-dual Weyl curvature $W^{+}$ identifies with $\Psi$ via $\Sigma$ whilst the scalar curvature is given by $R=4 \Lambda$, which gives us the Einstein constant: $\operatorname{Ric}\left(g_{\Sigma}\right)=\Lambda g_{\Sigma}$. In fact, there is a small subtlety here. Equations (2.6), 2.7) and (2.8) show that $(A,-\Sigma,-\Psi)$ is a critical point for the Plebanski action with $\Lambda$ replaced by $-\Lambda$. To resolve this ambiguity and determine which sign we should use, note that we fixed an orientation of the bundle $E$. Meanwhile, the bundle $\Lambda^{+}$is naturally oriented. We choose the sign of $\Sigma$ in order that the isomorphism $\Sigma: E \rightarrow \Lambda^{+}$is orientation preserving. With this choice of sign, $W^{+}=\Psi$ and $\operatorname{Ric}\left(g_{\Sigma}\right)=\Lambda g_{\Sigma}$.

\subsection{The pure connection action}

At this stage we must restrict to the case $\Lambda \neq 0$. The idea behind the pure connection formulation is that equations (2.7) and (2.8) can be used to eliminate $\Psi$ and $\Sigma$ and make the action a function purely of the connection $A$. To do this, we must begin with a connection $A$ which is definite (see Definition 1.4). Write $\mathscr{D}$ for the set of definite connections and $\mathscr{P}=\{(A, \Sigma, \Psi): \operatorname{Tr}(\Sigma \wedge \Sigma)>0\}$ for the domain of the Plebanski action. We will define a map $\theta: \mathscr{D} \rightarrow \mathscr{P}$ of the form $\theta(A)=\left(A, \Sigma_{A}, \Psi_{A}\right)$ with the following crucial property: $A$ is a critical point of $S_{P} \circ \theta$ if and only if $\theta(A)$ is a critical point of $S_{P}$. Once $\theta$ has been found, the pure connection action is defined by $S=S_{P} \circ \theta$.

We first explain how to define $\Sigma_{A}$ given $A \in \mathscr{D}$. To satisfy equations (2.7) and 2.8), we must find a wedge-orthogonal 2 -form which is also in the span of the curvature forms. Since $A$ is definite,

$$
F^{i} \wedge F^{j}=Q^{i j} \mu
$$

for some positive-definite matrix $Q^{i j}$ and positive 4 -form $\mu$. Let $Y$ be a square-root of $Q$. When $\Lambda>0$ we take $Y$ to be the positive square root, when $\Lambda<0$ we take $Y$ to be the negative square root. Scaling $\mu$, scales $Q$ and so $Y$; we fix the choice of scale 
by requiring that $\operatorname{Tr} Y=\Lambda$. We denote the corresponding volume form $\mu_{A}$ to make clear that it is determined by $A$. To ease the notation we write $X=Y^{-1}$. Set

$$
\Sigma_{i}=X_{i j} F^{j}
$$

The $E^{*}$-valued 2-form $\Sigma_{i} \otimes e^{i}$ is globally defined and we denote it by $\Sigma_{A} \in \Omega^{2}\left(M, E^{*}\right)$ to emphasise that it is determined by $A$. The point is that the use of $X$ ensures that $\Sigma_{A}$ is wedge-orthogonal, so 2.7 is satisfied. Moreover, the $\Sigma_{i}$ and the $F_{i}$ span the same subspace, so it will be possible to satisfy equation (2.8) by the correct choice of $\Psi_{A}$. Indeed given that $\Sigma=X F_{A}$, we have $F_{A}=Y \Sigma_{A}$ and so defining $\Psi_{A}$ via

$$
\Psi_{A}+\frac{\Lambda}{3} \mathrm{Id}=Y
$$

is the only way to satisfy 2.8 .

We now say a word about the sign ambiguity which arose in our discussion of the Plebanski action. The image of $\Sigma_{A}$ is a definite sub-bundle of $\Lambda^{2}$, which we denote by $\Lambda_{A}^{+}$. The map $\Sigma_{A}: E \rightarrow \Lambda_{A}^{+}$is an isomorphism of oriented bundles and it either preserves or reverses orientation. Recall that $\Sigma_{A}=X F_{A}$ and the sign of $X$ agrees with that of $\Lambda$. So switching the sign of $\Lambda$ switches the sign of $\Sigma_{A}$. Given a definite connection only one of the two signs of $\Lambda$ is appropriate to consider, that for which the corresponding $\Sigma_{A}$ is orientation preserving. This sign can be seen directly from the curvature of $A$ as follows.

Definition 2.6. Given a definite connection $A$ and an orientation on $E$, the curvature can be interpreted as an isomorphism $E \rightarrow \Lambda_{A}^{+}$of oriented bundles. In our local frame this is the map $e_{i} \mapsto F^{i}$. We call $A$ positive-definite if this map is orientation preserving and negative-definite if this map is orientation reversing. Note that changing the orientation on $E$ changes the sign of this map and so the sign of the definite connection is well-defined.

Positive-definite connections are those for which $\Sigma_{A}$ is orientation preserving when $\Lambda>0$. Negative-definite connections are those for which $\Sigma_{A}$ is orientation preserving when $\Lambda<0$. From now on, given $\Lambda$ we consider only definite connections with the same sign. When $g$ is Einstein with $R_{+}$definite the Levi-Civita connection on $\Lambda_{g}^{+}$is definite and its sign agrees with that of $R_{+}$.

We define $\theta: \mathscr{D} \rightarrow \mathscr{P}$ by $\theta(A)=\left(A, \Sigma_{A}, \Psi_{A}\right)$. By construction, $\left(A, \Sigma_{A}, \Psi_{A}\right)$ automatically solves two of the three Euler-Lagrange equations: $\partial S_{P} / \partial \Psi=0=$ $\partial S_{P} / \partial \Sigma$. It follows that $A$ is a critical point of $S_{P} \circ \theta$ if and only if $\theta(A)$ is a critical point of $S_{P}$. The remaining Euler-Lagrange equation $\mathrm{d}_{A} \Sigma_{A}=0$ is now an equation purely for the definite connection $A$.

Finally, we can write the action $S_{P} \circ \theta$ purely in terms of the connection. Using the relationship between $\Sigma_{A}, \Psi_{A}$ and $F_{A}$ we see that

$$
S_{P}(\theta(A))=\frac{1}{2} \int_{M} X_{i j} F^{i} \wedge F^{j}=\frac{\Lambda}{2} \int_{M} \mu_{A}=\frac{\Lambda}{2} \operatorname{vol}\left(M, g_{A}\right)
$$

where $g_{A}=g_{\Sigma_{A}}$ denotes the Riemannian metric determined by $\Sigma_{A}$ and hence $A$.

Definition 2.7 (See [8, [5]). Let $\Lambda \neq 0$ and $A$ be a definite connection of the same sign as $\Lambda$. We call

$$
S(A)=\frac{\Lambda}{2} \operatorname{vol}\left(M, g_{A}\right)
$$

the pure connection action of $A$.

We summarise this discussion in the following statement.

Theorem 2.8 (See [8, 5]). Fix $\Lambda \neq 0$ and let $A$ be a definite connection of the same sign. $A$ is a critical point of the pure connection $S$ action if and only

$$
\mathrm{d}_{A} \Sigma_{A}=0
$$

When this happens, $g_{A}$ is an Einstein metric with $R_{+}$definite and of the same sign as $\Lambda$. Moreover all Einstein metrics with $R_{+}$definite arise this way. 
We close this section with some remarks about the existence of definite connections. In [4] it is shown that if a definite connection exists on $M$, then $2 \chi(M)+3 \tau(M)>0$. This is "one half" of the Hitchin-Thorpe inequality, a necessary condition for the existence of an Einstein metric 6, 11. No other obstructions to the existence of definite connections are known. The only known compact examples of positive definite connections are on $S^{4}$ and $\mathbb{C P}^{2}$, given by deforming the corresponding Levi-Civita connections of the standard Einstein metrics there. Meanwhile, there are negative definite connections on manifolds on which it is currently unknown if there are Einstein metrics. See [4, 5] for examples of definite connections and open questions about them.

\subsection{The gauge group}

Let $\mathscr{G}: E \rightarrow E$ denote the group of all maps which send fibres of $E$ to fibres by linear isometries (i.e. bundle isomorphisms). We call $\mathscr{G}$ the gauge group. An element $\gamma \in \mathscr{G}$ covers a diffeomorphism on the base $M$ and this sets up an exact sequence

$$
1 \rightarrow \mathscr{G}_{0} \rightarrow \mathscr{G} \rightarrow \operatorname{Diff}(M) \rightarrow 1
$$

where $\mathscr{G}_{0}$ is the subgroup of gauge transformations which cover the identity on $M$, the "usual" gauge group one sees in traditional gauge theory.

$\mathscr{G}$ acts on the space $\mathscr{A}$ of all connections in $E$, by pull-back. It preserves the open set $\mathscr{D} \subset \mathscr{A}$ of definite connections. The map $A \mapsto g_{A}$, sending a definite connection to its corresponding metric, is equivariant with respect to the action of $\mathscr{G}$ on $\mathscr{D}$ and the action of $\operatorname{Diff}(M)$ on metrics. It follows that the pure connection action $S: \mathscr{D} \rightarrow \mathbb{R}$ is $\mathscr{G}$-invariant.

Given $\eta \in \operatorname{Lie} \mathscr{G}$ we write $L_{\eta} A$ for the infinitesimal action of $\eta$ at $A$. To compute $L_{\eta} A$ it is convenient to move to the principal bundle description. $P \rightarrow M$ denotes the principal $\mathrm{SO}(3)$-bundle of frames of $E$. $\mathscr{G}$ is then the subgroup of diffeomorphisms of $P$ which commute with the principal $\mathrm{SO}(3)$-action.The Lie algebra $\operatorname{Lie}(\mathscr{G})$ is a subalgebra of vector fields on $P$. A connection $A$ is an $\mathrm{SO}(3)$-equivariant connection 1 -form with values in $\mathfrak{s o}(3)$. In this picture, the infinitesimal action $L_{\eta} A$ of $\eta \in \operatorname{Lie}(\mathscr{G})$ at $A$ is simply the usual Lie derivative.

We now give a useful formula for $L_{\eta} A$. Use $A$ to split the vector field $\eta$ into vertical and horizontal parts. When $\eta \in \operatorname{Lie}(\mathscr{G})$, the vertical part corresponds to a section $\xi \in \Omega^{0}(M, \mathfrak{s o}(E))$; meanwhile, the horizontal part is the $A$-horizontal lift to $P$ of a vector field $v$ on $M$. By Cartan's formula,

$$
L_{\eta} A=\mathrm{d}\left(\iota_{\eta} A\right)+\iota_{\eta}(\mathrm{d} A)=\mathrm{d}_{A}(\xi)+\iota_{v} F_{A}
$$

(We consider here a right action of $\mathscr{G}$ on $\mathscr{A}, A \mapsto \gamma^{*} A$ whereas usually in gauge theory one considers the corresponding left action. This accounts for the difference in sign of the term $\mathrm{d}_{A}(\xi)$ here when compared with standard gauge theory literature.)

\section{The Hessian of the pure connection action}

In this section we compute the Hessian of the pure connection action $S$. Recall that $\mathscr{D}$ denotes the set of definite connections (an open set in the affine space of all connections). Given $A \in \mathscr{D}$ which is a critical point of $S$, the Hessian $D^{2} S$ of $S$ at $A$ is a symmetric bilinear form on $T_{A} \mathscr{D}=\Omega^{1}(M, \mathfrak{s o}(E))$.

Theorem 3.1. Let $A$ be a critical point of $S$ corresponding to an Einstein metric for which $R_{+}<0$. Then $D^{2} S(a, a) \geqslant 0$ with equality if and only if $a=L_{\eta} A$ for some $\eta \in \operatorname{Lie}(\mathscr{G})$.

It turns out to be easiest to compute the Hessian of $S$ by passing via Plebanski action. Recall that $\mathscr{P}=\{(A, \Sigma, \Psi): \operatorname{Tr}(\Sigma \wedge \Sigma)>0\}$ denotes the domain of Plebanski action. In the previous section we introduced a map $\theta: \mathscr{D} \rightarrow \mathscr{P}$ given by $\theta(A)=$ $\left(A, \Sigma_{A}, \Psi_{A}\right)$ where $\Sigma_{A}$ and $\Psi_{A}$ are defined by 2.9 and 2.10 . The pure connection 
action is the pull-back of Plebanski action by $\theta$. It follows that at a critical point $A$, the Hessian of the pure connection action is the pull-back by $\theta$ of the Hessian of $S_{P}$ at $\theta(A)$. To compute $D^{2} S$ we will begin with the Hessian of Plebanski action at an arbitrary critical point $(A, \Sigma, \Psi)$ (not necessarily of the form $\theta(A)$ for a definite connection $A$ ). Then we pull this back to $\mathscr{D}$ via $\theta$. To complete the proof of Theorem 3.1, we will also bring gauge fixing into the picture.

\subsection{The Hessian before gauge fixing}

We begin with the Hessian of the Plebanski action. Fix a critical point $(A, \Sigma, \Psi)$ of $S_{P}$ and choose a tangent direction $t=(a, \sigma, \phi)$. Write $D^{2} S_{P}(t, t)$ for the Hessian of $S_{P}$ in this direction. Differentiating the equations (2.3), (2.4) and (2.5) again with respect to $(a, \sigma, \phi)$ (and recalling our conventions 2.1) and (2.2) we see that $D^{2} S_{P}(t, t)$ is equal to

$$
\int_{M}\left\{-\epsilon_{i j}{ }^{k} a^{i} \wedge a^{j} \wedge \Sigma_{k}+2\left(\left(\mathrm{~d}_{A} a\right)^{i}-\phi^{i j} \Sigma_{j}\right) \wedge \sigma_{i}-\left(\Psi^{i j}+\frac{\Lambda}{3} \delta^{i j}\right) \sigma_{i} \wedge \sigma_{j}\right\}
$$

We now consider the Hessian of the pure connection action $S$. Let $A$ be a definite connection which is a critical point of $S$ so that $\theta(A)=\left(A, \Sigma_{A}, \Psi_{A}\right)$ is a critical point of $S_{P}$. Given an infinitesimal change $a$ of $A$, the Hessians of $S$ and $S_{P}$ are related by

$$
D^{2} S(a, a)=D^{2} S_{P}\left(\theta_{*}(a), \theta_{*}(a)\right)
$$

Write $\theta_{*}(a)=(a, \sigma, \phi)$ where $\sigma$ and $\phi$ denote the corresponding infinitesimal changes in $\Sigma_{A}$ and $\Psi_{A}$ respectively. To proceed we will need the precise dependence of $\sigma$ and $\phi$ on $a$. To obtain this note that the tangent vector $(a, \sigma, \phi)$ preserves the conditions (2.7) and 2.8. Differentiating (2.7) we see that

$$
\sigma_{i} \wedge \Sigma_{j}+\Sigma_{i} \wedge \sigma_{j}-\frac{2}{3}\left(\sigma_{k} \wedge \Sigma_{k}\right) \delta_{i j}=0
$$

Differentiating 2.8 gives

$$
\left(\mathrm{d}_{A} a\right)^{i}-\phi^{i j} \Sigma_{j}-Y^{i j} \sigma_{j}=0
$$

(Recall that $Y=\Psi_{A}+\frac{\Lambda}{3}$ Id.) Rearranging this gives

$$
\sigma_{i}=X_{i j}\left(\left(\mathrm{~d}_{A} a\right)^{j}-\phi^{j k} \Sigma_{k}\right)
$$

(Recall that $X=Y^{-1}$.) From here we can solve for $\phi$ in terms of $\mathrm{d}_{A} a$ via 3.2. We do this as follows.

Using the local frame $\Sigma^{j}$ for $\Lambda^{+}$we write the self-dual part of $\sigma_{i}$ as $\sigma_{i}^{+}=Z_{i}^{j} \Sigma_{j}$ for some $3 \times 3$-matrix valued function $Z$. Similarly, we put $\left(\mathrm{d}_{A}^{+} a\right)^{i}=N^{i j} \Sigma_{j}$, so 3.4 reads

$$
Z_{i}^{j}=X_{i k}\left(N^{k j}-\phi^{k j}\right)
$$

Now an arbitrary $3 \times 3$-matrix has three irreducible parts: the symmetric trace-free component, the skew component and the trace component. Equation $(3.2)$ says that the symmetric trace-free part of $Z$ is zero. This uniquely determines $\phi^{i j}$ in terms of $N^{i j}$ and $X_{i j}$. To see this write $L: S_{0}^{2} E \rightarrow S_{0}^{2} E$ for the linear bundle map $L(\phi)=S_{0}^{2}(X \phi)$ where $S_{0}^{2}$ denotes the projection operator sending a matrix to the trace-free symmetric part. Then $\phi$ must solve $L(\phi)=S_{0}^{2}(X N)$. This has a unique solution $\phi \in S_{0}^{2} E$ provided $L$ is injective. Suppose then that $L(\psi)=0$ for some $\psi \in S_{0}^{2} E$ or, in other words, that

$$
\psi+X \psi X^{-1}=f X^{-1}
$$

for some function $f$. Taking the trace and using the fact that $\operatorname{Tr} X^{-1}=\Lambda$ we see that $f=0$. On the one hand the eigenvalues of the similar matrices $\psi$ and $X \psi X^{-1}$ are equal, on the other hand the fact that $\psi=-X \psi X^{-1}$ shows the eigenvalues are opposite. The only conclusion is that $\psi=0$ and so $L$ is an isomorphism as required.

We can now give the formula for the Hessian of the pure connection action. 
Lemma 3.2. Let $A \in \mathscr{D}$ be a critical point of $S$ and let $a \in T_{A} \mathscr{D}$. The Hessian of $S$ in the direction a is given by

$$
D^{2} S(a, a)=\int_{M}\left[-\epsilon_{i j}{ }^{k} a^{i} \wedge a^{j} \wedge \Sigma_{k}+X_{i j}\left(\left(\mathrm{~d}_{A} a\right)^{i}-\phi^{i k} \Sigma_{k}\right) \wedge\left(\left(\mathrm{d}_{A} a\right)^{j}-\phi^{j k} \Sigma_{k}\right)\right]
$$

where $\phi \in S_{0}^{2} E$ is the unique solution to $S_{0}^{2}(X \phi)=S_{0}^{2}(X N)$ where $d_{A}^{+} a=N \Sigma$.

Proof. We have seen that $\theta_{*}(a)=(a, \sigma, \phi)$ where $\sigma$ and $\phi$ satisfy (3.4). We now substitute (3.4) into (3.1) and use the fact that

$$
X_{i j}\left(\Psi^{i j}+\frac{\Lambda}{3} \delta^{i j}\right)=X_{i j} Y^{i j}=\delta_{i}^{j}
$$

This gives the claimed expression for $D^{2} S(a, a)$. The condition on $\phi$ is the content of the discussion after (3.5)

\subsection{The Hessian after gauge fixing}

In this section we will explain how the formula of Lemma 3.2 for $D^{2} S(a, a)$ simplifies when we impose additional gauge fixing conditions on $a$. Before discussing this however we need a short digression. Given a wedge-orthogonal $\Sigma$, we can define a triple of almost complex structures $J_{i}$ by "raising an index" on $\Sigma_{i}$ with $g_{\Sigma}$. (It is for this reason that that the metric $g_{\Sigma}$ is scaled so that $\left|\Sigma_{i}\right|^{2}=2$.) Equivalently, one can define the action of $J_{i}$ on 1-forms via the equation

$$
J_{i}(\alpha)=*\left(\Sigma_{i} \wedge \alpha\right)
$$

One can check that the $J_{i}$ satisfy the quaternion relations:

$$
J_{i} J_{j}=\epsilon_{i j}{ }^{k} J_{k}-\delta_{i j}
$$

At this point we use crucially that $\Sigma: E \rightarrow \Lambda^{+}$is orientation-preserving; if it were orientation-reversing then $-J_{i}$ would satisfy $(3.7)$.

We will use the freedom to act by gauge transformations to ensure that the infinitesimal change in connection $a^{i}$ satisfies the following two conditions:

Definition 3.3. Let $A$ be a definite connection which is a critical point of the pure connection action $S$. Let $\Sigma_{A}$ be determined by $A$ via $2.9 p$. We say that $a \in \Omega^{1}(M, \mathfrak{s o}(E))$ is in horizontal gauge if

$$
\Sigma_{i} \wedge a^{i}=0
$$

In terms of the almost complex structures, this is equivalent to $J_{i} a^{i}=0$. Given $a$ in horizontal gauge, we say that it is also in vertical gauge if in addition:

$$
\epsilon^{i j}{ }_{k} \Sigma_{j} \wedge\left(\mathrm{d}_{A} a\right)^{k}=0
$$

When $a$ is in both horizontal and vertical gauge we say that $a$ is fully gauge fixed.

We briefly explain the names. Loosely speaking, gauge transformations are made up of diffeomorphisms on $M$ and purely "vertical" gauge transformations which preserve each fibre of $E$. The horizontal gauge fixing condition gives a complement to the infinitesimal action of diffeomorphisms, i.e. of horizontal lifts of vector fields from $M$. Now for those $a$ which are in horizontal gauge, the second condition $(3.9)$ is equivalent to Coulomb gauge, $\mathrm{d}_{A}^{*} a=0$, as the following lemma shows. This then gives a complement to the infinitesimal action of vertical gauge transformations.

Lemma 3.4. If $a$ is in horiztonal gauge and $\mathrm{d}_{A} \Sigma_{A}=0$ then

$$
\begin{aligned}
* a^{i} & =\epsilon_{k}^{i j} \Sigma_{j} \wedge a^{k} \\
\left(\mathrm{~d}_{A}^{*} a\right)^{i} & =-*\left(\epsilon^{i j}{ }_{k} \Sigma_{j} \wedge\left(\mathrm{d}_{A} a\right)^{k}\right)
\end{aligned}
$$


Proof. In terms of the almost complex structures $J_{i}$, the horizontal gauge condition (3.8) reads $J_{i} a^{i}=0$ or, equivalently, $a^{i}=-\epsilon^{i j k} J_{j} a_{k}$. Taking the Hodge star, and using the fact that $*^{2}=-1$ on 3 -forms, we obtain $* a^{i}=\epsilon^{i j}{ }_{k} \Sigma_{j} \wedge a^{k}$. Now, since $\mathrm{d}_{A} \Sigma_{A}=0$ and $\mathrm{d}_{A}^{*}=-* \mathrm{~d}_{A} *$ equation 3.11) follows from 3.10.

Before discussing how to enforce these two conditions, we show the effect they have on the Hessian of the pure connection action.

Proposition 3.5 (The gauge-fixed Hessian). Let $A \in \mathscr{D}$ be a critical point of the pure connection action $S$. Let a be an infinitesimal change in $A$ which satisfies both gauge fixing conditions (3.8) and (3.9). Then the Hessian of the pure connection action in this direction is given by

$$
D^{2} S(a, a)=\int_{M}\left(|a|^{2}-X_{i j}\left\langle\left(\mathrm{~d}_{A}^{-} a\right)^{i},\left(\mathrm{~d}_{A}^{-} a\right)^{j}\right\rangle\right) \mu_{A}
$$

In particular, when $R_{+}$is negative definite, so that $X$ is negative definite,

$$
D^{2} S(a, a) \geqslant 0
$$

with equality if and only if $a=0$.

Proof. Recall the formula for $D^{2} S_{A}(a, a)$ given in Lemma 3.2 above. When $a$ is in horizontal gauge, the first term in the integrand is

$$
-\epsilon_{i j}{ }^{k} a^{i} \wedge a^{j} \wedge \Sigma_{k}=a^{i} \wedge * a^{i}=|a|^{2} \mu_{A}
$$

We now turn to the second term in the integrand, namely

$$
X_{i j}\left(\left(\mathrm{~d}_{A} a\right)^{i}-\phi^{i k} \Sigma_{k}\right) \wedge\left(\left(\mathrm{d}_{A} a\right)^{j}-\phi^{j k} \Sigma_{k}\right)
$$

Here $\phi \in S_{0}^{2} E$ is determined by $a$ via $S_{0}^{2}(X \phi)=S_{0}^{2} X N$ where $\left(\mathrm{d}_{A}^{+} a\right)^{i}=N^{i j} \Sigma_{j}$. The Coulomb gauge condition (3.9) says that the skew-symmetric part of $N$ vanishes. Meanwhile, differentiating the horizontal gauge condition (3.8) and using $\mathrm{d}_{A} \Sigma_{A}=0$ gives

$$
\Sigma_{i} \wedge\left(\mathrm{d}_{A} a\right)^{i}=0
$$

which means that the trace of $N$ vanishes. It follows that $N$ is itself a symmetric trace-free matrix and so $\phi=N$. It follows that

$$
\left(\mathrm{d}_{A} a\right)^{i}-\phi^{i j} \Sigma^{j}=\left(\mathrm{d}_{A}^{-} a\right)^{i}
$$

is purely anti-self-dual. The second term is thus

$$
X_{i j}\left(\mathrm{~d}_{A}^{-} a\right)^{i} \wedge\left(\mathrm{d}_{A}^{-} a\right)^{j}
$$

For anti-self-dual forms, the wedge product is minus the pointwise inner product which completes the proof.

\subsection{Gauge fixing}

We now show that the space of fully gauge fixed $a$ defines a complement to the infinitesimal gauge action. Recall that elements $\eta \in \operatorname{Lie}(\mathscr{G})$ correspond to pairs $(v, \xi)$ where $\xi \in \Omega^{0}(M, \mathfrak{s o}(E))$ and $v$ is a vector field on $M$. The infinitesimal action of $\eta$ at $A$ is $L_{\eta}(A)=\iota_{v} F_{A}+\mathrm{d}_{A} \xi$

Let

$$
W=\left\{a \in C^{\infty}\left(\Lambda^{1} \otimes E\right): a \text { is fully gauge fixed, 3.8) and } 3.9 \text { hold }\right\}
$$

The goal of this section is to prove the following: 
Proposition 3.6. There is a direct sum decomposition

$$
\Omega^{1}(M, \mathfrak{s o}(E))=\left\{L_{\eta} A: \eta \in \operatorname{Lie}(\mathscr{G})\right\} \oplus W
$$

Before giving the proof we first try and lighten the notation a little. Write

$$
p: \Lambda^{1} \otimes \mathfrak{s o}(E) \rightarrow \Lambda^{1}, \quad p(a)=J_{i} a^{i}
$$

Recall that the horizontal gauge condition 3.8 is equivalent to $p(a)=0$. Write

$$
q: T M \rightarrow \Lambda^{1} \otimes \mathfrak{s o}(E), \quad q(v)=\iota_{v} F_{A}
$$

To prove Proposition 3.6 we must show that for any $a$ there is a unique solution $(v, \xi)$ to the pair of equations

$$
\begin{aligned}
p\left(a+q(v)+\mathrm{d}_{A} \xi\right) & =0 \\
\mathrm{~d}_{A}^{*}\left(a+q(v)+\mathrm{d}_{A} \xi\right) & =0
\end{aligned}
$$

We will show that this leads to an elliptic equation for $\xi$. As we will see shortly, the composition $p \circ q$ is invertible. Assuming this for the moment, for any choice of $a$ and $\xi$, there is a unique choice of $v$ solving (3.18), namely

$$
v=-(p \circ q)^{-1} \circ p\left(\left(\mathrm{~d}_{A} \xi\right)+a\right)
$$

Write

$$
\Pi=1-q \circ(p \circ q)^{-1} \circ p
$$

It is simple to check that $\Pi$ is a projection operator, projecting $\Lambda^{1} \otimes E$ onto $\operatorname{ker}(p)$ against $\operatorname{im}(q)$. In other words, $\Pi(a)$ is what one obtains by putting $a$ in horizontal gauge using the infinitesimal action of tangent vectors. Now substituting (3.20) into $(3.19)$ we see that $(3.18)$ and $(3.19)$ are equivalent to the following single equation for $\xi$ :

$$
\mathrm{d}_{A}^{*} \Pi \mathrm{d}_{A} \xi=-\mathrm{d}_{A}^{*} \Pi a
$$

We will prove that 3.21 has a unique solution in three steps: by showing that $\mathrm{d}_{A}^{*} \Pi \mathrm{d}_{A}$ is elliptic; that it has index zero and finally that it is injective.

The symbol of $\mathrm{d}_{A}^{*} \Pi \mathrm{d}_{A}$ in the direction of the covector $\alpha$ is the map

$$
\operatorname{Sym}(\alpha): E \rightarrow E, \quad \operatorname{Sym}(\alpha)(\xi)=\langle\alpha, \Pi(\alpha \otimes \xi)\rangle
$$

To compute this we first give a formula for $\Pi$. Along the way we show that $p \circ q$ is invertible. Recall that $F^{i}=Y^{i j} \Sigma_{j}$ where $Y=\Psi+\frac{\Lambda}{3}$ Id. Our curvature assumption that $R_{+}<0$ is equivalent to $Y<0$.

Lemma 3.7. We have $p \circ q(v)=-\Lambda v^{b}$ where $v^{b}$ denotes the covector which is metric dual to the tangent vector $v$. It follows that $(p \circ q)^{-1}(\alpha)=-\Lambda^{-1} \alpha^{\#}$ where $\alpha^{\#}$ is the tangent vector metric dual to the covector $\alpha$.

Proof. This is a direct calculation, using the quaternion relations (3.7).

$$
\begin{aligned}
p \circ q(v) & =J_{i}\left(\iota_{v} F^{i}\right) \\
& =J_{i}\left(Y^{i j} \iota_{v} \Sigma_{j}\right) \\
& =Y^{i j} J_{i} J_{j}\left(v^{b}\right) \\
& =Y^{i j}\left(\epsilon_{i j}{ }^{k} J_{k}\left(v^{b}\right)-\delta_{i j} v^{b}\right) \\
& =-\operatorname{Tr}(Y) v^{b} \\
& =-\Lambda v^{b}
\end{aligned}
$$


Lemma 3.8. The projection $\Pi$ has the following expression in terms of the self-dual curvature matrix $Y^{i j}$

$$
(\Pi a)^{i}=a^{i}+\Lambda^{-1} \epsilon_{k j}{ }^{l} Y^{i k} J_{l}\left(a^{j}\right)-\Lambda^{-1} Y^{i j} a^{j}
$$

Proof. Again, this is direct calculation.

$$
\begin{aligned}
(\Pi a)^{i} & =a^{i}-q \circ(p \circ q)^{-1} \circ p(a)^{i} \\
& =a^{i}+\Lambda^{-1} \iota\left(J_{j} a^{j}\right)^{\#} F^{i} \\
& =a^{i}+\Lambda^{-1} Y^{i k} \iota_{\left(J_{j} a^{j}\right) \#} \Sigma^{k} \\
& =a^{i}+\Lambda^{-1} Y^{i k} J_{k} J_{j}\left(a^{j}\right) \\
& =a^{i}+\Lambda^{-1} \epsilon_{k j}{ }^{l} Y^{i k} J^{l}\left(a^{j}\right)-\Lambda^{-1} Y^{i j} a^{j}
\end{aligned}
$$

Lemma 3.9. When $R_{+}$is negative definite, $\mathrm{d}_{A}^{*} \Pi \mathrm{d}_{A}$ is an elliptic operator on sections of $\mathfrak{s o}(E)$.

Proof. We use the above formula for $\Pi$ to compute the symbol acting on the covector $\alpha$. Write $\xi=\xi^{i} \otimes \widehat{e}_{i}$.

$$
\begin{aligned}
\operatorname{Sym}(a)(\xi) & =\langle\alpha, \Pi(\alpha \otimes \xi)\rangle \\
& =\xi^{i}|\alpha|^{2}+\Lambda^{-1} \epsilon_{k j}{ }^{l} \xi^{j} Y^{i k}\left\langle\alpha, J_{l}(\alpha)\right\rangle-\Lambda^{-1} Y^{i j} \xi^{j}|\alpha|^{2} \\
& =\left(\xi^{i}-\Lambda^{-1} Y^{i j} \xi^{j}\right)|\alpha|^{2}
\end{aligned}
$$

where in the last line we have used that $\alpha$ and $J_{l}(\alpha)$ are orthogonal. It follows that, for $\alpha \neq 0$, the kernel of $\operatorname{Sym}(\alpha)$ is exactly the space of eigenvectors of $Y$ with eigenvalue $\Lambda$. But $\Lambda=\operatorname{Tr}(Y)$ and all eigenvalues of $Y$ are strictly negative, so none can be equal to $\Lambda$. It follows that $\operatorname{Sym}(\alpha)$ is invertible and so $\mathrm{d}_{A}^{*} \Pi \mathrm{d}_{A}$ is elliptic.

Lemma 3.10. When $R_{+}$is negative definite, the index of $\mathrm{d}_{A}^{*} \Pi \mathrm{d}_{A}$ is zero.

Proof. If $\Pi$ were self adjoint then $\mathrm{d}_{A}^{*} \Pi \mathrm{d}_{A}$ would be also and it would follow immediately that the index vanishes. This is not the case however because, in general, $\operatorname{ker} p$ and $\operatorname{im} q$ are not orthogonal subspaces. To remedy this, consider the path $Y_{t}^{i j}=t \Psi^{i j}+\frac{\Lambda}{3} \delta^{i j}$ of endomorphisms and the corresponding path $F_{t}^{i}=Y_{t}^{i j} \Sigma^{j}$ of sections of $\Lambda^{2} \otimes \mathfrak{s o}(E)$. The forms $F_{t}$ define a path of maps $q_{t}: T M \rightarrow \Lambda^{1} \otimes \mathfrak{s o}(E)$ given by $q_{t}(v)=\iota_{v} F_{t}$. Since $Y=Y_{1}$ is negative-definite, the same is true for $Y_{t}$ for all $t \in[0,1]$. Moreover, $\operatorname{Tr} Y_{t}=\Lambda$. These were the only properties we used in the above arguments. It follows that $\Pi=1-q_{t} \circ\left(p \circ q_{t}\right)^{-1} \circ p$ is a projection of $\Lambda^{1} \otimes \mathfrak{s o}(E)$ onto ker $p$ against $\operatorname{im} q_{t}$ and, moreover, that $\mathrm{d}_{A}^{*} \Pi_{t} \mathrm{~d}_{A}$ is a path of elliptic operators. It follows that our operator has the same index as $\mathrm{d}_{A}^{*} \Pi_{0} \mathrm{~d}_{A}$. But when $t=0$, im $q_{0}$ is the orthogonal complement of $\operatorname{ker} p$ and so $\Pi_{0}$ is a self-adjoint projection. It follows that the index of $\mathrm{d}_{A}^{*} \Pi_{0} \mathrm{~d}_{A}$, and hence of all the $\mathrm{d}_{A}^{*} \Pi_{t} \mathrm{~d}_{A}$, vanishes for $t \in[0,1]$.

Proposition 3.11. When $R_{+}$is negative-definite, the map

$$
\mathrm{d}_{A}^{*} \Pi \mathrm{d}_{A}: C^{\infty}(E) \rightarrow C^{\infty}(E)
$$

is an isomorphism.

Proof. Now that we know $\mathrm{d}_{A}^{*} \Pi \mathrm{d}_{A}$ is elliptic of index zero, if suffices to show that it is injective. Suppose then that $\mathrm{d}_{A}^{*} \Pi \mathrm{d}_{A} \xi=0$. This means that $\Pi \mathrm{d}_{A} \xi$ is fully gauge fixed. By Proposition 3.5 it follows that either $\Pi_{A} \xi=0$, or the Hessian in this direction is non-zero. Now, by definition of $\Pi$, there exists $v \in C^{\infty}(T M)$ such that $\Pi \mathrm{d}_{A} \xi=\mathrm{d}_{A} \xi+\iota_{v} F$. In other words, $\Pi \mathrm{d}_{A} \xi$ is pure gauge. Since the pure connection action is gauge invariant, the Hessian vanishes in pure gauge directions. It follows that $\Pi \mathrm{d}_{A} \xi=0$ or, in other words, that $\iota_{v} F=-\mathrm{d}_{A} \xi$. 
Now the infinitesmial gauge transformation $\xi$ doesn't change the induced metric $g_{A}$ and so $v$ is a Killing field. But the metric has negative Ricci curvature and so $v$ must vanish 1 . It follows that $\mathrm{d}_{A} \xi=0$. From this we see that $F_{A}(\xi)=0$, or in other words that $Y^{i j} \xi^{j}=0$. Since $Y$ is invertible we obtain $\xi=0$ as required.

This Proposition, together with the discussion immediately after equations 3.18 and (3.19), completes the proof of Proposition 3.6. which shows that, on the linear level, one can always fully gauge fix $a$ via the infinitesimal action of the gauge group.

\subsection{The proof of Theorem 3.1}

We now give the proof of Theorem 3.1. Let $A$ be a critical point of the pure connection action $S$ and assume that the corresponding Einstein metric has $R_{+}<0$. Let $a$ be an infinitesimal deformation of $A$. By Proposition 3.6 we can write $a=b+L_{\eta} A$ for $b$ fully gauge-fixed and $\eta \in \operatorname{Lie}(\mathscr{G})$. Since $S$ is $\mathscr{G}$ invariant, $D^{2} S\left(L_{\eta} A, \cdot\right)=0$. It follows that $D^{2} S(a, a)=D^{2} S(b, b)$. Theorem 3.1 now follows from Proposition 3.5 .

\section{The proof of local rigidity}

In this section we show how Theorem 3.1 implies our main result Theorem 1.2 . We give the statement again for convenience.

Theorem. Let $g$ be an Einstein metric on a compact orientable 4-manifold $M^{4}$, with either $R_{+}<0$ or $R_{-}<0$. Then $g$ is locally rigid.

Proof. Let $h$ be an infinitesimal Einstein deformation of $g$. We must find a vector field $v$ on $M$ for which $L_{v} g=h$. The idea of the proof is to show that the metric deformation $h$ is induced by a deformation $a$ of the definite connection $A$ corresponding to $g$ and, moreover, with $a \in \operatorname{ker} D^{2} S$. This will imply that $a$ is pure gauge and so the same will be true of $h$.

It is certainly not true that an arbitrary metric deformation can be achieved by deforming the connection. This can be seen by counting degrees of freedom. The space of connections is modelled on $\Omega^{1}(M, \mathfrak{s o}(E))$ and so has functional dimension 12 . The gauge group $\mathscr{G}$ meanwhile has functional dimension 7 , so one can only expect to reach a space of metrics with effective functional dimension 5 . Meanwhile metrics have functional dimension 10 of which diffeomorphisms account for 4 dimensions, leaving effective functional dimension 6 which is larger. This means we must use in an essential way the fact that $h$ is an infinitesimal Einstein deformation.

Write $\mathscr{M}$ for the space of Riemannian metrics and $G: \mathscr{D} \rightarrow \mathscr{M}$ for the map which sends a definite connection to the corresponding Riemannian metric. This map is equivariant for the action of $\mathscr{G}$ on $\mathscr{D}$ and $\operatorname{Diff}(M)$ on $\mathscr{M}$. Let $A \in \mathscr{D}$ be the critical point of the pure connection action $S$ with $G(A)=g$. We seek $a \in T_{A} \mathscr{D}$ such that $D_{A} G(a)=h$ and with $a \in \operatorname{ker} D^{2} S$. From here, Theorem 3.1 shows that $a=L_{\eta} A$ for some $\eta \in \operatorname{Lie}(\mathscr{G})$. Writing $v \in C^{\infty}(M, T M)$ for the pushforward of $\eta$ to $M$, equivariance of $G$ then implies that $h=L_{v} g$.

To proceed let $\widehat{\Sigma}(t): E \rightarrow \Lambda^{2}$ be a path of wedge-orthogonal 2-forms with $\widehat{\Sigma}(0)$ corresponding to $A$ via 2.9 . Write the corresponding path of metrics as

$$
\widehat{g}(t)=g_{\widehat{\Sigma}(t)}
$$

and choose $\widehat{\Sigma}(t)$ so that $\widehat{g}^{\prime}(0)=h$.

Now let $A(t)$ be the pull-back to $E$ of the $\widehat{g}(t)$-Levi-Civita connection on $\Lambda^{+}(\widehat{g}(t))$ via $\widehat{\Sigma}(t)$. By construction $A(0)=A$. Each connection $A(t)$ is again definite (at least

\footnotetext{
${ }^{1}$ We mention the simple proof of this standard fact for completeness. If $v$ is Killing, Bochner's formula gives $\frac{1}{2} \Delta|v|^{2}=|\nabla v|^{2}-\operatorname{Ric}(v, v)$. When $\operatorname{Ric}<0$ and the manifold is compact, integrating this equality shows $v$ must vanish.
} 
for small $t$ ). Write $a=A^{\prime}(0) \in T_{A} \mathscr{D}$. $A(t)$ determines a wedge-orthogonal map $\Sigma(t)=\Sigma_{A(t)}$ via 2.9 and a path of metrics $g(t)=g_{A(t)}$.

It is important to note that $g(t)$ need not be equal to the path $\widehat{g}(t)$ we first started with. We will prove that when $h$ is an infinitesimal Einstein deformation, that these paths agree to first order: $g^{\prime}(0)=\widehat{g}^{\prime}(0)$. Write $\sigma=\Sigma^{\prime}(0)$ and $\widehat{\sigma}=\widehat{\Sigma}^{\prime}(0)$. In fact, we will show that $\sigma=\widehat{\sigma}$, from which it follows that $g^{\prime}(0)=\widehat{g}^{\prime}(0)$.

The 2-form $\widehat{\Sigma}(t)$ is wedge-orthogonal. Differentiating this condition with respect to $t$ and evaluating at $t=0$ we get

$$
\widehat{\sigma}_{i} \wedge \Sigma_{j}+\Sigma_{j} \wedge \widehat{\sigma}_{i}-\frac{2}{3}\left(\widehat{\sigma}_{k} \wedge \Sigma_{k}\right) \delta_{i j}=0
$$

(Cf. equation (3.2).) Next write

$$
F_{A(t)}=B(t)+C(t)
$$

where $B(t)$ is $\widehat{g}(t)$-self-dual and $C(t)$ is $\widehat{g}(t)$-anti-self-dual. Since $\widehat{g}(0)$ is Einstein and $h$ is an infinitesimal Einstein deformation, $C(t)=O\left(t^{2}\right)$. We write (locally) $B^{i}=\hat{Y}^{i j}(t) \widehat{\Sigma}_{j}(t)$. At $t=0, \widehat{Y}(0)=Y$, the matrix representative for $R_{+}$of the original Einstein metric $\widehat{g}(0)=g$. Symmetries of the Riemann tensor ensure that $\widehat{Y}(t)$ is symmetric for all $t$. Moreover, since $h$ is an infinitesimal Einstein deformation, $\operatorname{Tr} \widehat{Y}=\Lambda+O\left(t^{2}\right)$. If follows that $\widehat{Y}^{\prime}(0)$ is trace-free. Differentiating $F_{A(t)}$ with respect to $t$ and evaluating at $t=0$ we get

$$
\left(\mathrm{d}_{A} a\right)^{i}=\left(\widehat{Y}^{\prime}\right)^{i j}(0) \Sigma_{i}+Y^{i j} \widehat{\sigma}_{j}
$$

Meanwhile, the path $A(t)$ of definite connections determines a path $(\Sigma(t), \Psi(t))$ via 2.9$)$ and 2.10$)$. The tangent $(a, \sigma, \phi)$ to the whole path $\left(A, \Sigma_{A}, \Psi_{A}\right)(t)$ of Plebanski variables at $t=0$ satisfies the equations (3.2) and (3.3). Moreover, given $a$, we saw that there are unique $\sigma$ and $\phi$ solving $(3.2)$ and $(3.3)$. But these equations are identical to (4.1) and 4.2 satisfied by $\widehat{\sigma}$, with $\phi$ replaced by $\widehat{Y}^{\prime}(0)$. It follows that $\sigma=\hat{\sigma}$ as claimed (and $\phi=\widehat{Y}^{\prime}(0)$ but we will not use this).

Finally we have to check that the tangent vector $a \in T_{A} \mathscr{D}$ is in $\operatorname{ker} D^{2} S$. This is equivalent to $a$ being tangent to the critical locus of $S$ which is in turn equivalent to $\mathrm{d}_{A(t)} \Sigma(t)=O\left(t^{2}\right)$. Recall that $A(t)$ is the pull-back of the Levi-Civita connection via $\widehat{\Sigma}(t)$. It follows from Lemma 2.2 that $\mathrm{d}_{A(t)} \widehat{\Sigma}(t)=0$ for all $t$. But we have just seen that $\widehat{\Sigma}(t)=\Sigma(t)+O\left(t^{2}\right)$ and so $\mathrm{d}_{A(t)} \Sigma(t)=O\left(t^{2}\right)$ and $a \in \operatorname{ker} D^{2} S$ as claimed.

\section{References}

[1] Besson, G., Courtois, G., Gallot, S. Entropies et rigidité des espaces localement symétriques de courbure strictement négative, Geom. Funct. Anal. 5:5 (1995) 731-799.

[2] Biquard, O. Polycopié on Differential Geometry and Global Analysis, Unpublished lecture notes, https://www.math.ens.fr/ biquard/dgga2007.pdf

[3] Fine, J. A gauge theoretic approach to the anti-self-dual Einstein equations, Preprint, https://arxiv.org/abs/1111.5005

[4] Fine, J., Panov, D. Symplectic Calabi-Yau manifolds, minimal surfaces and the hyperbolic geometry of the conifold, J. Differential Geom., 82:1 (2009) 155-205.

[5] Fine, J., Krasnov, K., Panov, D. A gauge theoretic approach to Einstein 4-manifolds, New York J. Math., 20 (2014) 293-324.

[6] Hitchin, N.J. Compact four-dimensional Einstein manifolds, J. Differential Geom., 9 (1974) 435-441.

[7] Koiso, N. Non-deformability of Einstein metrics, Osaka J. Math. 15 (1978) 419433. 
[8] Krasnov, K. Pure connection action principle for general relativity, Phys. Rev. Lett. 106:25 (2011) 4pp.

[9] LeBrun, C. Einstein metrics and Mostow rigidity, Math. Res. Lett. 2 (1995) $1-8$.

[10] Plebanski, J.F. On the separation of Einsteinian substructures, J. Math. Phys. 18:12 (1977) 2511-2520.

[11] Thorpe, J.A. Some remarks on the Gauss-Bonnet integral, J. Math. Mech., 18 (1969) 779-786.

[12] Weinstein, A. Fat bundles and symplectic manifolds, Adv. in Math., $37: 3$ (1980) $239-250$. 